\title{
Fusion of HepG2 cells with mesenchymal stem cells increases cancer-associated and malignant properties: An in vivo metastasis model
}

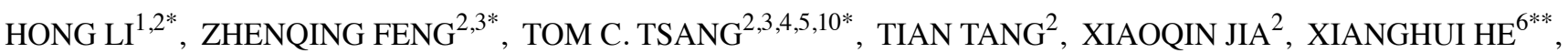 \\ MICHAEL E. PENNINGTON ${ }^{4 * *}$, MICHAEL S. BADOWSKI ${ }^{7}$, ANNA K.M. LIU $^{7}$, DEYU CHEN ${ }^{8}$, \\ DAVID T. HARRIS ${ }^{5,7}$, JESSE MARTINEZ ${ }^{5,9}$ and LINDA C. MEADE-TOLLIN ${ }^{4 * *}$ \\ ${ }^{1}$ Department of Pathology, Binzhou Medical University Hospital, Binzhou 256603; ${ }^{2}$ Key Laboratory of \\ Antibody Technique of the Ministry of Health, Department of Pathology, Nanjing Medical University, Nanjing 210029; \\ ${ }^{3}$ Jiangsu Key Laboratory of Cancer Biomarkers, Prevention and Treatment, Cancer Center, Nanjing Medical University, \\ Nanjing 210029, P.R. China; ${ }^{4}$ Cure Cancer Worldwide Corporation, Tucson, AZ 85728-5833; ${ }^{5}$ Arizona Cancer Center, \\ University of Arizona, Tucson, AZ 85721, USA; ${ }^{6}$ Department of General Surgery, Tianjin General Surgery Institute, \\ General Hospital of Tianjin Medical University, Tianjin 300052, P.R. China; ${ }^{7}$ Department of Immunobiology, \\ University of Arizona, Tucson, AZ 85724, USA; ${ }^{8}$ Department of Oncology, The Affiliated Hospital of \\ Jiangsu University, Zhenjiang, Jiangsu 212001, P.R. China; ${ }^{9}$ Department of Cell Biology and Anatomy, \\ University of Arizona, Tucson, AZ 85721, USA; ${ }^{10}$ Clinical Oncology Laboratory, Changzhou Tumor Hospital \\ Affiliated to Soochou University, Changzhou, Jiangsu 213002, P.R. China
}

Received January 29, 2014; Accepted February 21, 2014

DOI: $10.3892 / o r .2014 .3264$

\begin{abstract}
In the present study, we have tested the hypothesis that fusion between an altered cell and a mesenchymal stem cell produces a hybrid cell with enhanced characteristics associated with metastatic cancer cells, and we have developed a flexible model for investigating the mechanisms of metastasis. Human HepG2 cells with low metastatic potential were induced to fuse with rat bone marrow mesenchymal stem cells, and the progeny were compared with the parental cells for possession of enhanced in vitro and in vivo characteristics of malignant cells. Compared to the parental cells, the fused cells exhibited enhanced expression of E-cadherin, vimentin, Twist, Snail, matrix metalloproteinase 2 and 9 activities, aneuploidy and enhanced in vitro invasion and migration. In an in vivo xenograft assay, the fused cells generated increased numbers of metastatic liver and lung lesions. This model system is a flexible tool for investigation of the mechanisms of stem cell
\end{abstract}

Correspondence to: Dr Zhenqing Feng, Key Laboratory of Antibody Technique of the Ministry of Health, Nanjing Medical University, 140 Hanzhong Road, Nanjing 210029, P.R. China

E-mail: fengzhenqing@njmu.edu.cn

*Contributed equally; ** Contributed equally

Abbreviations: rt, room temperature; s-f, serum-free; MSC, mesenchymal stem cell; MMP, matrix metalloproteinase

Key words: stem cells, fusion, metastasis, invasion, in vivo models, aneuploidy fusion in carcinogenesis and metastasis and for the discovery of new therapeutic targets to inhibit metastasis.

\section{Introduction}

Numerous factors involved in the most deadly consequences of carcinogenesis and metastasis, are poorly understood. Interactions between motile stem cells and other cell types have been proposed to contribute to the mechanisms of carcinogenesis and acquisition of metastatic ability. Cell-specific in vivo models to explore these mechanisms could provide valuable insights into potential therapeutic targets. A well-established multi-step hypothesis has emerged from characterization of the hallmarks and genetic interactions associated with cancer; that accumulation of specific cellular genetic mutations can lead to carcinogenesis, malignancy and metastasis $(1,2)$. Although mutations surely play a role in carcinogenesis, clearly the initiation and progression of cancer involves more than genetic alterations alone. Other critical factors include the tumor microenvironment, inflammation (3), interactions with tumor stromal cells (4), [including myofibroblasts (5)] and recruitment of mesenchymal stem cells (MSCs) to the tumor microenvironment $(6,7)$. The recent discoveries of stem cells and cancer stem cells and investigations of their potential roles in cell fusion, carcinogenesis and metastasis have led to novel perspectives and approaches concerning the mechanisms of metastasis.

He et al (8) proposed a stem cell fusion model in which altered pre-malignant cells (including benign tumor cells) fuse with bone marrow-derived MSCs, to form a hybrid cancer cell with the hallmarks of metastatic cancer (2), and expressing other common phenotypes associated with malignancy. This 
hypothesis suggested that cell fusion and gene mutations are both important components of comprehensive carcinogenesis mechanisms. The hypothesis provided explanations for: i) the remarkable similarities between malignant cells and stem cells; ii) the ability of non-mutagens to be carcinogens; iii) the ability of non-mutagenic processes, such as wound healing or chronic inflammation, to promote malignant transformation and iv) the generation of aneuploidy and other common characteristics of malignant cancer cells. The observation of spontaneous or induced fusion of highly motile macrophages, monocytes, tissue stem cells or bone marrow-derived mesenchymal cells with differentiated or tumor cells, yielding hybrid cells with altered properties, has been reported (9). The fusion of various types of cells as a driver of metastatic cancer has been discussed in recent excellent reviews including Lu and Kang (10,11), Bjerkvig et al (12), and Pawelek and Chakraborty (13).

In the present study, we performed in vitro and in vivo experiments to explore the acquisition of enhanced malignant characteristics by cells derived from the polyethylene glycol (PEG)-induced fusion of rat bone marrow-derived MSCs and HepG2 cells. To distinguish between the parental cells, rat MSCs were labeled with DiI, and HepG2 cells were labeled by transfection with a plasmid expressing enhanced green fluorescent protein. The resulting dual-labeled fused progeny cells were distinguished from parental cells and collected by flow cytometry. Several phenotypic characteristics of malignant cells [aneuploidy, in vitro migratory and invasive ability, epithelial-mesenchymal transition (EMT) markers and regulatory factors, matrix metalloproteinase (MMP)2 and 9 activity and in vivo formation of metastases] were compared in the parental and fused cells. The experimental observations reported in this study support our previously proposed mechanism and the role of the fusion of stem cells with altered cells in the development of metastatic ability.

\section{Materials and methods}

Mesenchymal stem cell isolation, culturing and identification. Ten male Sprague-Dawley rats, 1 month in age, (Experimental Animal Center, Nanjing Medical University Center) with a body mass of $80-100 \mathrm{~g}$ were sacrificed and sterilized in $75 \%$ ethanol for $10 \mathrm{~min}$. Bone marrow was isolated under sterile conditions from femurs and tibias as previously described (14). To select a purified population of MSCs, briefly, bones were isolated and marrow was flushed out with serum-free (s-f) L-DMEM and filtered through a $0.1-\mu \mathrm{m}$ filter. Cells were propagated by incubation in standard growth medium. Non-adherent cells were removed after 4 and $24 \mathrm{~h}$ and every 2-3 days thereafter by gently washing with medium. Cultures were passed at $80-90 \%$ confluency 3-4 times by detachment with $0.25 \%$ trypsin for $1 \mathrm{~min}$. Flow cytometry was performed on trypsin-detached cells after washing and re-suspension with phosphate-buffered saline (PBS) 3 times. Antibody reagents for cell surface markers, including CD34, CD45, CD90 and CD105 (eBioscience, USA) were prepared according to the manufacturer's instructions and added to separate cell suspensions. The mixtures were incubated $20 \mathrm{~min}$ in the dark at room temperature (rt). The presence of specific cell markers was identified by flow cytometric analysis.
DiI labeling of MSC membranes. Confluent monolayers of MSCs were labeled for $3 \mathrm{~h}$ at $37^{\circ} \mathrm{C}$ with $400 \mu \mathrm{g}$ of DiI (Beyotime, China) in $20 \mathrm{ml}$ Dulbecco's modified Eagle's medium (DMEM), following the manufacturer's instructions. DiI is a lipophilic carbon cyanine dye characterized by specificity, high sensitivity, and strong fluorescence on binding to plasma membranes, facilitating the observation of whole cells. The labeled cells were washed in PBS and used in the cell fusion protocol.

HepG2 cell culture and transfection. Human HepG2 cells (Shanghai Institutes for Biological Sciences, CAS) have a low metastatic phenotype. They were cultured in high glucose DMEM (H-DMEM, 4.5 g/l glucose; Gibco, USA), 10\% fetal bovine serum (FBS), $2 \mathrm{mM}$ L-glutamine and $100 \mathrm{U} / \mathrm{ml}$ penicillin/streptomycin. The cells were transfected with pEGFP-N1 plasmid, which encodes a gene for green fluorescent protein (HepG2-eGFP). Transfected cells were selected for 4 weeks in $500 \mu \mathrm{g} / \mathrm{ml} \mathrm{G} 418$ (Invitrogen, USA). The fluorescence, monitored every 3 days, was retained during the selection and subsequent experiments. Then the HepG2-eGFP cells were identified and collected by flow cytometry.

Cell fusion and selection. A modification of the procedure of Köhler and Milstein was used (15). Briefly, 5x10 $0^{5}$ MSCs were mixed with $1 \times 10^{5}$ HepG 2 cells before washing 3 times by re-suspension in $30 \mathrm{ml} \mathrm{s}-\mathrm{f}$ medium and centrifugation for $5 \mathrm{~min}$ at 1,000 rpm at rt. One milliliter of polyethylene glycol, PEG2000 (Sigma, USA) was slowly added to the washed cell pellet. Thirty milliliters of s-f DMEM was added to the cellPEG2000 mixture after $1 \mathrm{~min}$. The suspension was incubated in a $37^{\circ} \mathrm{C}$ water bath for $10 \mathrm{~min}$ to allow fusion. The cells were pelleted, re-suspended in serum-containing DMEM, plated in standard growth medium, cultured 3 days, and sorted by flow cytometry to identify and collect the fused cells containing both GFP and DiI. Fused cells in culture retained their viability for 1 month. The cell fusion protocol used in this report is illustrated in Fig. 1A.

Chromosome analysis. Log phase HepG2-eGFP cells, MSCs and fused cells, suspended in s-f medium, were treated with $0.2 \mu \mathrm{g} / \mathrm{ml}$ colchicine and incubated for $3 \mathrm{~h}$. The cells were then treated with $75 \mathrm{mM} \mathrm{KCl}$ for $30 \mathrm{~min}$ in a $37^{\circ} \mathrm{C}$ water bath, fixed with methanol:acetic acid (3:1) mixture for $1 \mathrm{~h}$ at $\mathrm{rt}$, dried on pre-cooled slides and stained with Giemsa solution (Invitrogen). Ten mitotic images were selected for each cell type, and the number of chromosomes in each image was counted using an inverted microscope (Olympus) with a x10 objective and a X100 oil immersion lens. The average chromosome number/cell was calculated for each cell type at 2 and 4 weeks after fusion.

Assays of cellular invasion and migration. Assays were performed in triplicate in Transwell dishes with $8 \mu \mathrm{m}$ pore size inserts. Standard growth medium containing $20 \%$ FBS was placed in the bottom chambers of the Transwell dishes. Migrating or invading cells were counted at x100 magnification. One-way ANOVA was used to calculate p-values for the cell counts.

Migration assays. A suspension of 20,000 HepG2 cells, MSCs or fused cells in s-f DMEM was layered on uncoated 
inserts seated in Transwell dishes (Millipore, USA). Dishes were incubated for $16 \mathrm{~h}$ at $37^{\circ} \mathrm{C}$. The medium was removed from the upper and bottom chambers. The inserts were fixed $1 \mathrm{~h}$ with $4 \%$ paraformaldehyde and stained with $0.1 \%$ crystal violet at rt. Cells remaining on top of the insert were removed by scraping. Seven randomly selected fields of the invaded cells on the bottom of the insert were chosen and the number of invasive cells was counted.

Invasion assays. Transwell inserts were coated with $45 \mu \mathrm{l}$ of a 1:8 dilution of Matrigel (Becton-Dickinson, USA) in s-f DMEM and incubated for $1 \mathrm{~h}$ to gel. A suspension of 40,000 cells in s-f DMEM was layered onto the inserts as described above and incubated for 36-48 h. The invading cells on the bottom of the inserts were stained, and seven randomly selected microscopic fields were counted.

Western blot assay. The fused cells, HepG2 cells (included for comparison with the HepG2-eGFP cells), HepG2-eGFP cells and MSCs were harvested and lysed on ice for $10 \mathrm{~min}$ in RIPA lysis buffer (50 mM Tris-HCl, pH 7.5, $150 \mathrm{mM} \mathrm{NaCl,} 0.2 \mathrm{mM}$ EDTA, $1 \mathrm{mM}$ phenylmethylsulfonyl fluoride, $1 \% \mathrm{NP}-40$ and $0.25 \%$ deoxycholate; Beyotime, China). Protein concentration was assayed with a BCA assay kit (Pierce, USA) according to the manufacturer's instructions. The assay was as previously described (16) with the following modifications. Fifty micrograms of proteins from each cell type were denatured $5 \mathrm{~min}$ at $95^{\circ} \mathrm{C}$ in sample buffer $(250 \mathrm{mM}$ Tris, $\mathrm{pH} 6.8,10 \%$ SDS, $50 \%$ glycerol, $5 \%$ mercaptoethanol and $0.5 \mathrm{mM}$ bromphenol blue) and separated by electrophoresis on 10\% SDS-PAGE gels. Equiloading of samples was detected with a 1:1,000 dilution of $\beta$-actin antibody (Cell Signaling), as per the manufacturer's protocol. Proteins were transferred onto PVDF membranes (Millipore). Membranes were blocked with 5\% fat-free milk at $\mathrm{rt}$ for $2 \mathrm{~h}$ and cut. Sections were then incubated at $4^{\circ} \mathrm{C}$ overnight with a 1:1,000 dilution of one of the following primary monoclonal antibodies: anti-vimentin (GeneTex), anti-Ecadherin, anti-Twist1, anti-Snail and with anti- $\beta$-actin (all from Cell Signaling) at $4^{\circ} \mathrm{C}$. After three washes for $10 \mathrm{~min}$ in PBS supplemented with $0.05 \%$ Tween-20 (PBST), the membrane was incubated with a peroxidase-conjugated secondary antibody (Zhongshan Golden Bridge Biotechnology) for $2 \mathrm{~h}$ at $\mathrm{rt}$. Enhanced chemiluminescence reagent (Pierce) was used for detection as per the manufacturer's instructions. Gel images were scanned into a file and processed with PowerPoint software.

Gelatin zymography assays. For each of the three cell types, $2 \times 10^{5}$ cells/well in a 12-well plate were incubated for $12 \mathrm{~h}$ at $37^{\circ} \mathrm{C}$. The medium was removed, and $500 \mu \mathrm{l} \mathrm{s}-\mathrm{f}$ DMEM was added to each well. After incubation for $24 \mathrm{~h}$, the medium from each plate was harvested and pooled and separated by electrophoresis on a $10 \%$ SDS-PAGE gel containing $1 \mathrm{mg} / \mathrm{ml}$ gelatin to detect MMP2 and 9 activities. After electrophoresis, the gels were equilibrated in $2.5 \%$ Triton $\mathrm{X}-100$ and incubated in renaturing buffer $[50 \mathrm{mM}$ Tris- $\mathrm{HCl}(\mathrm{pH} 7.5), 10 \mathrm{mM} \mathrm{CaCl}$, $150 \mathrm{mM} \mathrm{NaCl}, 1 \mathrm{mM} \mathrm{ZnCl}_{2}$ and $0.02 \% \mathrm{NaN}_{3}$ ] for $42 \mathrm{~h}$ at $37^{\circ} \mathrm{C}$. The gel was stained with Coomassie R250 and destained until clear bands of MMP activity were visible against the dark blue background. Activities of MMP2 and 9 were identified by comparing band mobility with molecular weight standards.
Images were obtained by scanning gels with Alpha Innotech gel imaging systems.

Xenograft assay. Six-week-old BALB/c nude mice were purchased from Shanghai Laboratory Animal Company. Mice were maintained in a pathogen-free environment, under temperature-controlled conditions. Cages, bedding and drinking water were autoclaved and changed regularly. Food was sterilized by irradiation. Mice were maintained at a daily cycle of 12-h periods of alternating light and dark.

The fused cells, HepG2 cells and MSCs were harvested, counted and centrifuged. For each cell type, $2.4 \times 10^{7}$ cells were suspended in $240 \mu \mathrm{l}$ of Matrigel (Becton-Dickinson), and placed on ice. Twenty-one BALB/c nude male mice were randomly divided into three groups of seven mice, one group for each cell type. Before inoculation, all mice were anesthetized by intraperitoneal injection with $1 \%$ pentobarbital sodium (10 $\mu \mathrm{l} / \mathrm{g}$ body weight) (Sigma, Germany). The peritoneal cavity was opened and the left liver lobe was exposed. The left liver lobe of each mouse was injected with $30 \mu \mathrm{l}$ of Matrigel containing $3 \times 10^{6}$ cells. Implantation was finished within $6 \mathrm{~h}$. The mice received gentamicin in drinking water $(80,000 \mathrm{U} / \mathrm{l})$ up to 1 week following implantation. Body mass and survival rate were calculated each week (data not shown). Surviving mice were sacrificed after 10 weeks and necropsied to assess metastatic tumor formation. The livers, lungs, kidneys and brains of mice in each group were isolated.

Histological preparation of the specimens. The liver, lung, brain and kidney were removed and fixed in $10 \%$ formalin, dehydrated and embedded in paraffin. Sections of 4- $\mu \mathrm{m}$ thickness were cut and stained with hematoxylin and eosin $(\mathrm{H} \& \mathrm{E})$ for microscopic observation by a pathologist.

\section{Results}

Fusion of HepG2-eGFP cells with DiI-labeled MSCs generates dual-labeled progeny cells. MSCs were selected from the total population of bone marrow-derived cells based upon their differential adherent properties in culture (14). Since MSCs do not express a unique marker on their surfaces, their identification often involves screening for multiple surface markers such as CD34, CD45, CD90 and CD105. Flow cytometric analysis of MSCs showed that cell surface markers were characteristically positive for CD90 and CD105 and characteristically negative for CD34 and CD45 (data not shown) indicating that MSCs were not contaminated by hematopoietic cell lineages. As liver cancer cells can express some of the same CD markers as MSCs, we labeled the MSCs with DiI and HepG2 cells with the plasmid eGFP.

Rates of spontaneous fusion can vary between 1 in $10^{2}$ to 1 in $10^{6}$ in vitro (9) and in vivo (17). Flow cytometric analysis, gated to select for only dual labeled cells, detected both labels in $6.9 \%$ of the total cell population after fusion was induced by PEG2000 treatment, an indicator of the efficiency of fusion (Fig. 1D). The sorted fused cells were collected and cultured. They remained viable until use in the experiments 2 weeks later. The fused cells retained their GFP plasmidinduced green fluorescent properties for at least 1 month after fusion. DiI red fluorescence labeling was maintained in cultures 

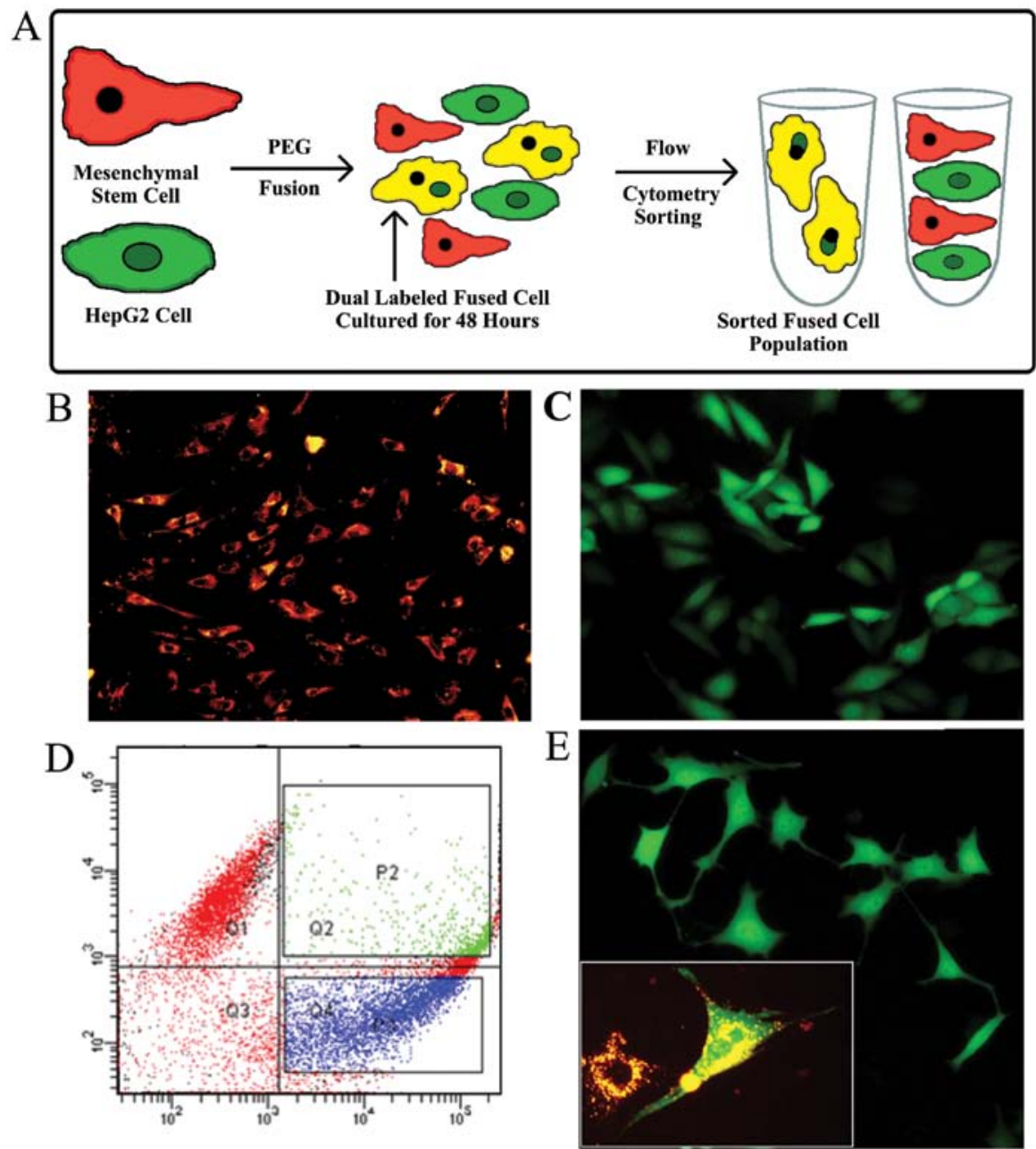

Figure 1. Experimental sequence. (A) Polyethylene glycol-mediated cell fusion of DiI-labeled mesenchymal stem cells (MSCs) and eGFP-transfected HepG2 cells. Fluorescent images were captured before sorting of parental cells: (B) DiI-labeled MSCs; (C) eGFP-transfected HepG2 cells. (D) Flow cytometry of cells after fusion. Four quadrants were defined: quadrant 1, DiI fluorescence; quadrant 2, cells with dual fluorescence. P2 represents the dual-labeled fused cells which were collected. Quadrant 3, cells with low fluorescence. Quadrant 4, cells with only GFP fluorescence. (E) Fused mononuclear cells imaged one month after FACS sorting, exhibiting mesenchymal cell morphology (larger panel) and a fused cell with two nuclei (inset).

for $\sim 1$ week. Fluorescence microscopy of both types of labeled progenitor cells [(Fig. 1B and C, and the fusion hybrid 1 month after fusion (Fig. 1E)], showed homogeneous labeling. In the Fig. 1E insert image of the fused cells, cells with more than one nucleus are visible. Many fused cells contained two nuclei initially, but after 36-48 h, contained only one nucleus. The marked mesenchymal morphology of long cellular extrusions, shallow cell bodies, and prominent nuclei observed in Fig. 1E, suggest that an EMT occurred.

Fused cells are aneuploids. If fusion occurs, the fused cells should initially contain more chromosomes per cell than either progenitor cell type. The average numbers of Giemsa-stained chromosomes/cell were compared for each type at 2 and 4 weeks after fusion, and are shown in Table I. The average chromosome count was markedly increased in the fused cells, and was not a multiple of the haploid chromosome number. This observation is characteristic of aneuploidy, and indicates that the cells contained chromosomes obtained from both MSCs and HepG2-GFP cells. There was no extensive loss of chromosomes in the progenitor cells over one month, the dura-
Table I. Average number of chromosomes.

\begin{tabular}{lccc}
\hline Cells & $\begin{array}{c}\text { Two weeks } \\
\text { after fusion }\end{array}$ & $\begin{array}{c}\text { Four weeks } \\
\text { after fusion }\end{array}$ & Change (\%) \\
\hline MSCs & $42.3 \pm 1.1$ & $41.3 \pm 4.1$ & -0.02 \\
HepG2-GFP & $86.0 \pm 8.4$ & $89.6 \pm 16.0$ & +0.04 \\
Fused cells & $157.5 \pm 17.5$ & $128.9 \pm 21.3$ & -18.20 \\
\hline
\end{tabular}

The average chromosomal number/cell for each cell line and the fused progeny cells was determined at 2 and 4 weeks as described in Materials and methods. Standard deviations of the mean are indicated. The fused cells have a significantly greater alteration in the average number between 2 and 4 weeks in culture than the parental cells. MSCs, mesenchymal stem cells.

tion of observation, but the average chromosome counts for fused cells decreased $18 \%$ between 2 and 4 weeks, indicating that some chromosomal instability was present. 
A Migration through transwell chamber-FBS coat

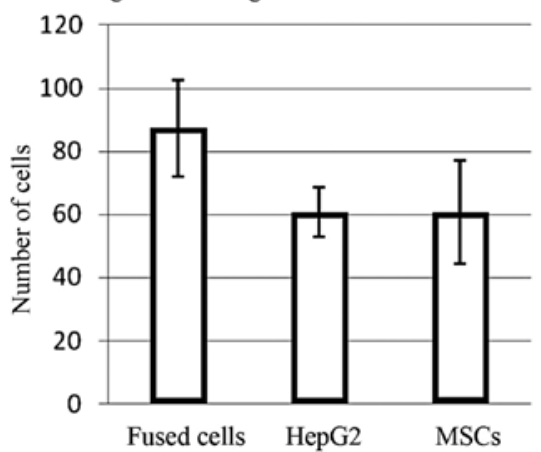

B Invasion through matrigel-transwell chamber

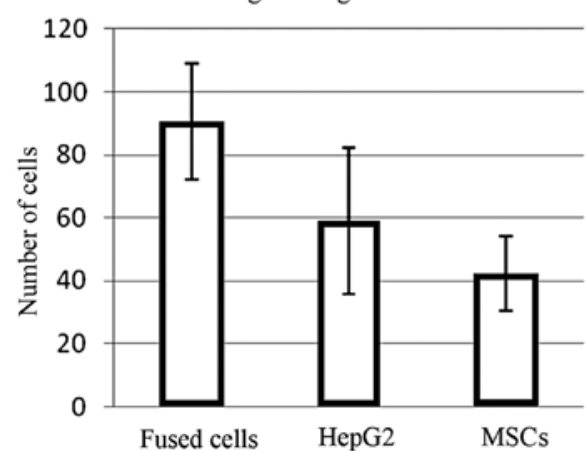

Figure 2. Fused cells exhibit enhanced mobility compared to HepG2 cells. (A) Comparison of cell migration ability through 8- $\mu \mathrm{m}$ Transwell chambers after $16 \mathrm{~h}$. Error bars represent the standard deviation of the results obtained. Fused cells exhibited increased motility compared to that of the HepG2 cells $(\mathrm{p}=0.0003)$ and the MSCs $(\mathrm{p}=0.001)$. (B) Invasion through Matrigel-coated Transwell inserts after $48 \mathrm{~h}$. Fused cells exhibited increased invasive ability compared to the HepG2 cells $(\mathrm{p}=0.047)$ and the MSCs $(\mathrm{p}=0.002)$. MSCs, mesenchymal stem cells.

A

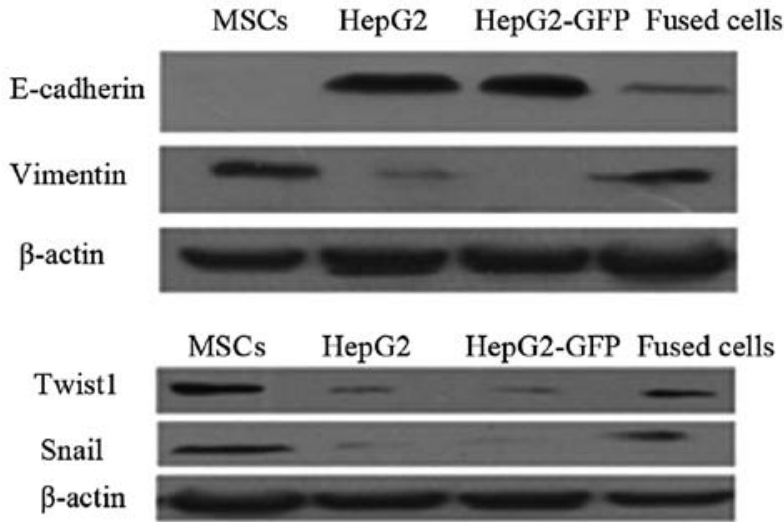

$\mathrm{C}$

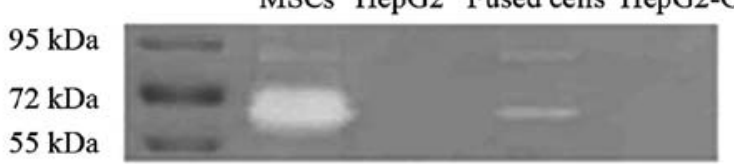

Figure 3. Fused cells express EMT markers and exhibit MMP activity (A and B) Western blot assays of whole cell lysate E-cadherin, vimentin, Twist and Snail. $\beta$-actin levels were detected to monitor equivalent loading of protein. (C) Gelatin zymograms demonstrating that fused cells secrete increased MMP2 and MMP9 activity when compared to HepG2 cells. EMT, epithelial-mesenchymal transition; MMP, matrix metalloproteinase.

Fused cells exhibit increased migration and invasion. The number of fused cells migrating through the uncoated Transwell membranes was $\sim 50 \%$ greater than the numbers of the MSCs or HepG2 cells ( $\mathrm{p}=0.015431$ and 0.000613 , respectively) (Fig. 2A). The fused cells were also $\sim 50 \%$ more invasive through Matrigel-coated Transwell membranes than the HepG2 cells, and twice as invasive as the MSCs ( $\mathrm{p}=0.014211$ and 0.007125 , respectively) (Fig. 2B). Error bars represent the standard deviation of the cell number obtained for each field counted.

Fused cells exhibit increased EMT marker expression and increased levels of active MMP2 and MMP9. After 1 month in culture, the fused cells exhibited morphology typical of mesenchymal cells, unlike their progenitors (Fig. 1E). Epithelial cells undergoing EMT typically lose expression of epithelial markers such as E-cadherin and increase expression of mesenchymal markers including vimentin. Western blot assays detected reduced expression of E-cadherin and increased expression of vimentin in the fused cells when compared to HepG2 cells (Fig. 3A). The EMT regulatory factors Twist1 and Snail were also highly expressed in the fused cells and MSCs, when compared to levels in the HepG2/ HepG2-eGFP cells (Fig. 3B). Gelatin zymography detected higher activities of secreted MMP2 and MMP9 in the fused cells when compared with the activity levels in the HepG2 and HepG2-eGFP cells (Fig. 3C).

Fused cells are more metastatic in vivo than MSCs or HepG2 cells. The fused cells, HepG2-eGFP cells and MSCs were orthotopically injected into the left liver lobe of three separate groups of nude mice. After 10 weeks, the survival rate was $28.58 \%$ in the fusion group and $57.14 \%$ in the HepG2-eGFP group.

Gross examination. One hepatocellular carcinoma lesion at the site of orthotopic injection occurred in the left liver lobe of each mouse of the fusion group and the HepG2-eGFP group. Malignant hepatocellular carcinoma lesions were observed around the orthotopic lesion in the injected liver lobes (Fig. 4A and B). The average number of liver malignant lesions was $4.50 \pm 1.29$ in the fusion group, and was $2.0 \pm 0.82$ in the HepG2-eGFP group.

Metastatic carcinoma lesions were present in the lungs of the fusion group and in the HepG2 group. The number of liver metastases showed a statistically significant difference between the 2 groups (Fig. $4 \mathrm{C})(\mathrm{p}<0.05)$. No lesions were found in the livers and lungs of the mice inoculated with the MSCs.

Histological examination. Histological staining and microscopic observation of sections of liver from the mice inoculated with fused cells revealed a hepatocellular carcinoma invading normal liver tissue. At high magnification, a few residual hepatocytes were noted in the carcinoma tissue (Fig. 4D). However, in tissue sections from the HepG2-eGFP-inoculated group, a well-defined hepatocellular carcinoma was observed. Fibrous 


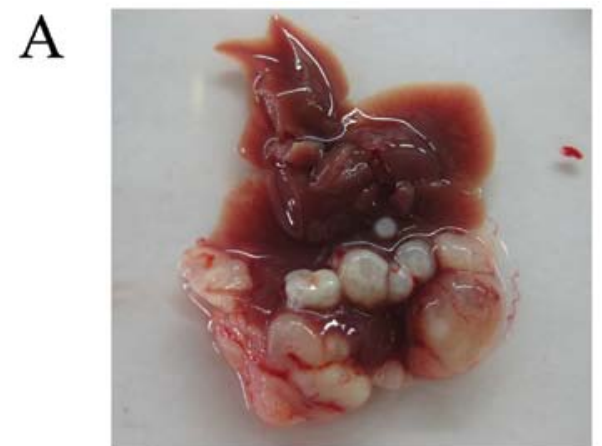

Fused Cells

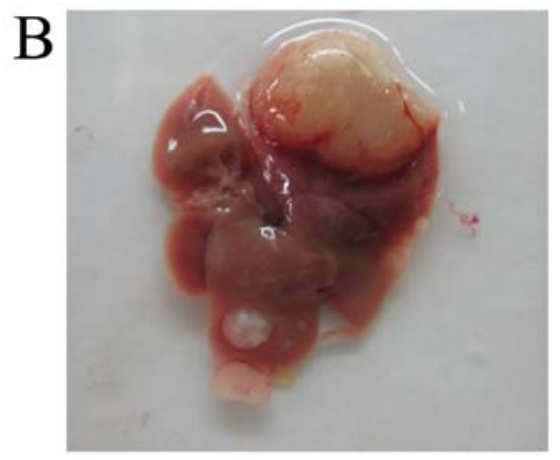

HepG2
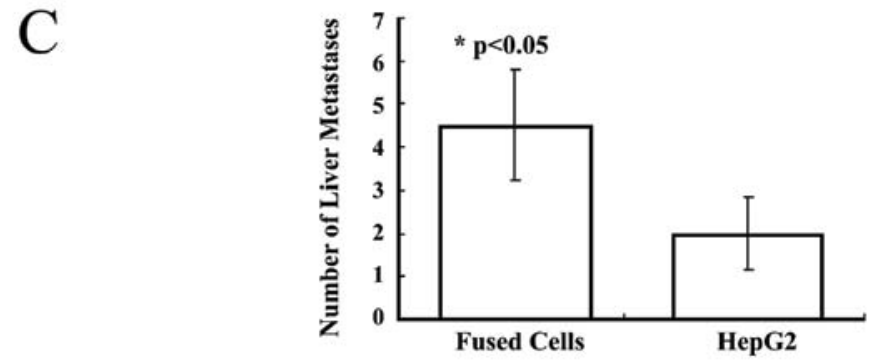

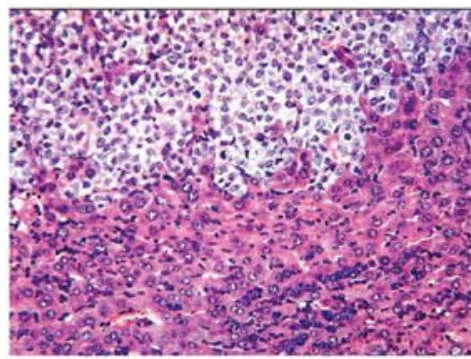

Fused Cells

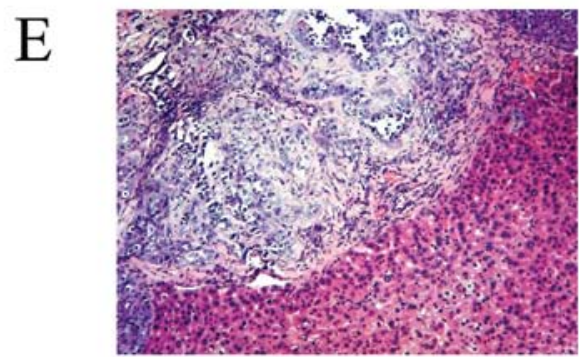

HepG2

Figure 4. Orthotopic injection of fused cells produces multiple undifferentiated hepatic tumors. Three million cells suspended in Matrigel were injected into the left hepatic lobe of each of 7 nude mice/cell type. Representative examples of gross hepatic tumors formed by (A) fused cells and (B) HepG2 cells are shown. (C) Comparison of the number of hepatic tumors/liver by gross examination of livers from 4 surviving mice/group. Hepatic tumors stained with $\mathrm{H} \& \mathrm{E}$ reagent, x100 magnification: (D) fused cells; (E) HepG2 cells. HepG2 cells formed well-differentiated noninvasive tumors. Fused cell tumors were poorly differentiated and invasive. H\&E, hematoxylin and eosin.

connective tissue surrounded the hepatocellular carcinoma, and it did not invade into the normal liver tissue (Fig. 4E).

Many lung metastatic lesions were observed. Representative lung tissue sections from fused cells (Fig. 5A) and HepG2 cells (Fig. 5B) are shown. The average number of lung metastases present was $3.29 \pm 1.89$ per pulmonary lobe in the tumors generated by the fused cells, and was $1.25 \pm 0.75$ in the HepG2-eGFP inoculated mice. As shown in Fig. 5C, the numbers of metastases detected in lung tissue from mice inoculated with the parental and progeny cells are compared. A total of 20 metastatic lesions were detected in lungs of the mice inoculated with fused cells compared to 5 detectable lesions generated in the mice inoculated with the HepG2 cells. No metastases were found in the kidney or brain in any mice. All organs were normal in the mice receiving MSCs. These results indicate that the fusion between the HepG2-eGFP cells and MSCs enhanced tumor metastasis in vivo.

\section{Discussion}

It has been argued that the introduction of wild-type tumor suppressors into a recipient cell by fusion should reverse the loss of heterozygosity present in the recipient cell which caused a more malignant phenotype, and should thus, make the recipient cell less transformed and less malignant. The stem cell fusion hypothesis (8) predicts the opposite, that the fused cells would be both more migratory and more invasive. To test this hypothesis, we fused HepG2 low-tumorigenic cancer cells and MSCs and compared the fused cells to each of the parental cell types. These initial experiments support our hypothesis, and indicate that despite our use of cells from two different species to generate the fused cells for our in vivo experiments, the mechanisms of generation of metastatic tumorigenic cells may have common features in different species. Although additional characterization and identification of rat and human DNA in the tumors from fused cells should be conducted, the clear differences in the properties of the parental and progeny cells in these initial experiments indicate that those differences are not simply due to the contamination of fused progeny cells with the HepG2 parent. In most comparisons described in this report, the fused cells possessed more malignancy-associated factors than either parental cell type. Similarly, fusing human gastric epithelial GES-1 cells with human umbilical cordderived MSCs was found to result in malignant transformation 


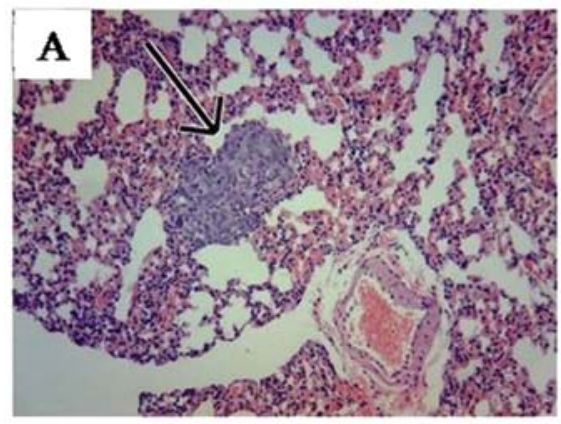

Fused cells

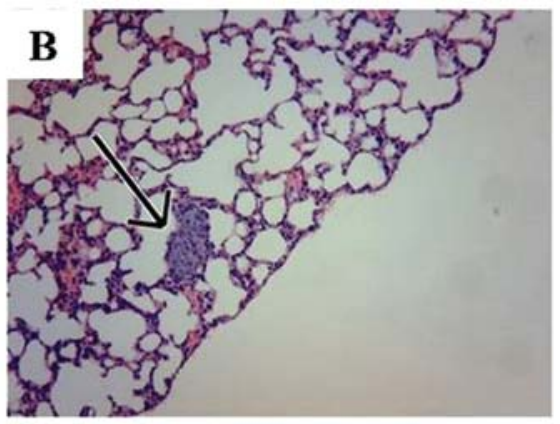

HepG2

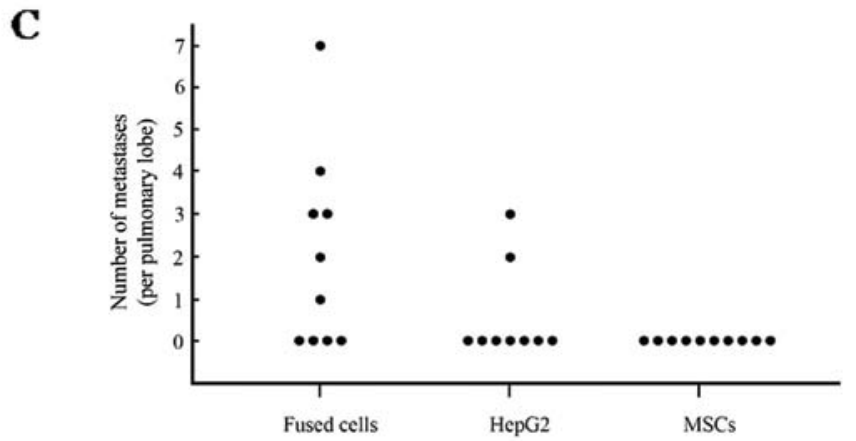

Figure 5. Fused cells generate an increased number of metastatic lesions in the lung. Lungs were harvested from 2 mice/group. Metastases were counted in a representative H\&E section from each of the 5 pulmonary lobes. A representative field is shown. (A) Fused cells; (B) HepG2 cells. The arrows indicate the presence of small metastatic lesions. (C) Graphical analysis of lung metastases. Fused cells generated 20 total lesions, compared to 5 total lesions generated by the HepG2 cells. H\&E, hematoxylin and eosin.

of the progeny cells (unpublished data, personal communication from Dr Xianghui He).

Aneuploidy is commonly observed in solid tumor cells, and is considered a hallmark of malignancy. It is also a predicted consequence of the stem cell fusion hypothesis. Numerous reviews and articles have presented data and theories supporting aneuploidy as a cause of cancer [for example (18)]. Aneuploidy may not always be associated with a pathological state. Aneuploid cells are found in the embryonic brain (19) and in the liver (20), and do not seem to always create detrimental effects. Several studies (21) support the variability and complexity of the effects and interactions of cellular aneuploidy, chromosomal instability, the presence of other mutations that suppress proliferation (such as p53), or amplify the cellular stresses induced by aneuploidy. Although it has been often suggested that aneuploidy is the event driving the ultimate pre-cancerous to metastatic conversion, the possibility that fusion actually drives the aneuploidy has received less attention. The aneuploidy we observed in the fused cells was maintained for at least 1 month, even though nuclear fusion was observed in the cultured fused cell population after $<1$ month. The $18 \%$ decrease in the average number of chromosomes after 1 month in the fused cells is indicative of chromosomal instability. In fact, an earlier application of the stem cell fusion model by Stein-Werblowsky and Ablin (22) demonstrated hyperchromasia in prostate cancer cells as a consequence of fusion of spermatozoa with normal prostatic epithelial cells. Our findings in this report support the predictions of the cell fusion model, and suggest many additional experiments which could clarify the relationship between fusion, aneuploidy, cancer progression and metastasis.
Since the cultured fused cells had irregular morphology and protruding processes, characteristics of cells undergoing EMT, we compared the expression of markers associated with EMT, a developmental process in which epithelial cells reduce intercellular adhesion and acquire mesenchymal properties, including downregulation of the epithelial marker E-cadherin and upregulation of the mesenchymal markers vimentin, Twist and Snail (23). In certain tumors, EMT results from tumor cell-MSC-induced fusion (24). Our western blot assays of fused cell total protein detected a decrease in expression of E-cadherin and an increase in expression of vimentin, supporting the occurrence of an EMT. In addition, both Twist and Snail were highly expressed in the fused cells. These alterations in EMT markers are also associated with invasion and metastasis. The matrix metalloproteinases (MMPs) are a family of ECM-degrading enzymes involved in EMT and cellular migration and invasion. Several MMPs, including MMP2 and MMP9, are induced in HepG2 cells by Snail (25). MMP9 transcription and cellular invasion are induced by overexpression of Snail in MDCK cells (26). Increased expression of Snail and secretion of active MMP2 and 9 were observed in the fused cells.

The lack of adequate in vivo models presents a major challenge to both basic research understanding of metastasis and therapeutic development $(27,28)$. Fusion between genetically altered cells and stem cells and their use in a tumorigenic and metastatic in vivo assay can be a flexible approach to the development of specific genetically defined and diverse models of metastasis. A recent report described specific genetic alterations of normal human lung epithelial cells which transformed them into tumorigenic cells (29). These cells were tumorigenic, 


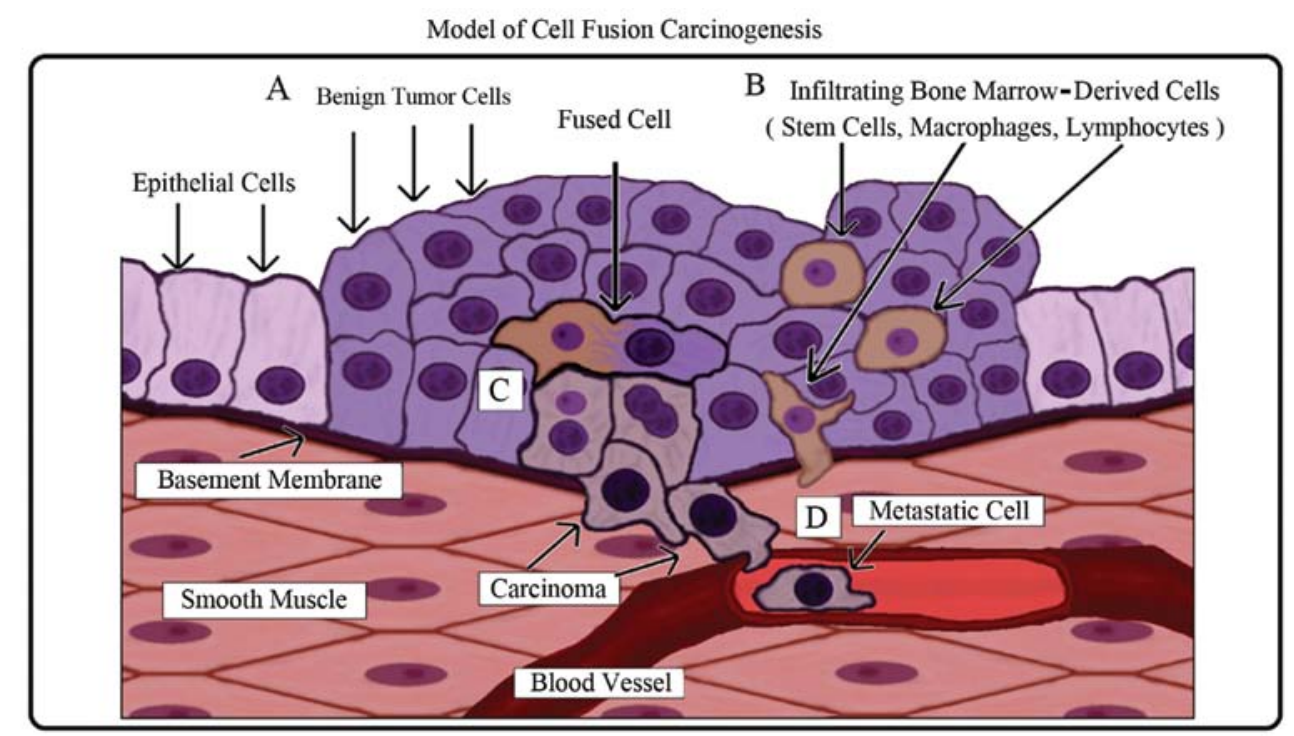

Figure 6. Model of cell fusion carcinogenesis. (A) In carcinogenesis, genetic alterations in normal cells result in benign hyperplasia and local tissue damage. (B) Bone marrow-derived cells (stem cells, macrophages and lymphocytes), are recruited to damaged tissue. (C) Fusion between an altered cell and stem cell forms a metastatic carcinoma cell. (D) Epigenetic traits inherited from the stem cell produce a metastatic cell.

but not metastatic in a mouse model. The observations made in this in vivo xenograft assay with orthotopic injection of fused cells into livers of nude mice establish further support for an increased metastatic phenotype in fused cells. Increased local invasiveness of the fused cells compared to the HepG2 cells was manifested as more numerous liver tumors as well as more metastatic lung lesions. Multiple models consisting of different cell types with specific genetic alterations fused with stem cells could be developed and evaluated using the Materials and methods described in this manuscript. Such model systems may allow for more accurate screening and testing of therapeutic targets for prevention of the most deadly consequence of carcinogenesis: acquisition of metastatic capacity.

A schematic of the model is illustrated in Fig. 6. Carcinogenesis is a multi-step process in which genetic mutations can induce an altered hyperplastic cell phenotype which then forms a benign neoplasm and subsequent local tissue damage (Fig. 6A). Bone marrow-derived cells (stem cells, macrophages and lymphocytes) are recruited to repair the damaged tissue (Fig. 6B). In the process of normal repair, fusion between an altered cell and a stem cell occurs (Fig. 6C). The resulting hybrid cell acquires epigenetic properties from the stem cell such as self-renewal, plasticity, and the capability to migrate to and survive in circulation (metastasis) while retaining both epithelial characteristics and mutations from the original tumor cell (Fig. 6D)

The fusion event is a potential mechanism for transferring stem cell-like properties to the altered cell. It is important to note that traits and qualities of metastatic cells overlap significantly with those of stem cells. This is no coincidence and its importance cannot be overstated. The individual mechanistic elements imparting 'stemness' may include a variety of protein, RNA mediated and/or epigenetic controls. However, regardless of the molecular mechanism(s) responsible, the stem cell may be a wellspring from which the altered cell perverts stem cell potency into metastatic activity.
These experiments were undertaken to test the previously proposed hypothesis that fusion between an altered, potentially premalignant cell, with little or no metastatic ability, and a bone marrow-derived mesenchymal stem cell, could form a hybrid cell with increased metastatic capacity and increased levels of phenotypic markers associated with cancer cells. We demonstrated that i) viable hybrid cells can be generated from fusion of a human liver cancer tumor cell line and rat MSCs, and identified by a double fluorescent label approach. ii) Fused liver-MSC hybrid cells, when orthotopically inoculated into nude mice, formed more tumors at both the liver injection site and at a distant site, the lung, than did control cells from either parental type. iii) The fused cells, compared to parental cells, were aneuploid, expressed higher levels of two matrix metalloproteinases associated with invasion and metastasis, expressed higher levels of markers and regulatory factors associated with an epithelial to mesenchymal transition, and exhibited an enhanced motility in Transwell assays, all phenotypes characteristic of malignant cells. iv) In histological comparisons of representative sections of the nodules formed by fused cells or the liver cancer cell line, tumors arising after fused cell inoculation invaded normal tissue, while those formed after inoculation of the low-metastatic HepG2 cells did not. v) We propose that this system is a very adaptable model for investigation of the mechanisms of metastasis. Specifically, altered cells with known genotypes when fused with MSCs, would yield multiple systems for investigation. We are continuing to expand and develop this approach to the generation of much needed, specific and useful models of metastasis.

\section{Acknowledgements}

We thank Yong Li, Xialing Zhang and Yuhua Li for assistance with this project. We are grateful to Drs G. Tim Bowden, Rein Kilkson and Richard J. Ablin for their teachings, and to our colleagues Drs Yu Duan, Jian Wang and Amy H.T. Davis for their helpful discussions. We also appreciate Clifton 
Leaf's book for continuing to inspire us. This study was supported by Cure Cancer Worldwide Company of USA and the Natural Science Foundation of Shandong Province (no. ZR2013HQ018).

\section{References}

1. Cairns J: Cancer, Science and Society. Freeman, San Francisco, 1978.

2. Hanahan D and Weinberg RA: Hallmarks of cancer: the next generation. Cell 144: 646-674, 2011.

3. Guthrie GJ, Charles KA, Roxburgh CS, Horgan PG, McMillan DC and Clarke SJ: The systemic inflammation-based neutrophillymphocyte ratio: experience in patients with cancer. Crit Rev Oncol Hematol 88: 218-230, 2013.

4. Casazza A, Di Conza G, Wenes M, Finisguerra V, Deschoemaeker S and Mazzone M: Tumor stroma: a complexity dictated by the hypoxic tumor microenvironment. Oncogene: Apr 22, 2013 (Epub ahead of print). doi: 10.1038/onc.2013.121.

5. De Wever O, Demetter P, Mareel M and Bracke M: Stromal myofibroblasts are drivers of invasive cancer growth. Int J Cancer 123: 2229-2238, 2008.

6. Mishra PJ, Mishra PJ, Glod JW and Banerjee D: Mesenchymal stem cells: flip side of the coin. Cancer Res 69: 1255-1258, 2009

7. Hass R and Otte A: Mesenchymal stem cells as all-round supporters in a normal and neoplastic microenvironment. Cell Commun Signal 10: 26, 2012.

8. He X, Tsang TC, Pipes BL, Ablin RJ and Harris DT: A stem cell fusion model of carcinogenesis. J Exp Ther Oncol 5: 101-109, 2005.

9. Ding J, Jin W, Chen C, Shao Z and Wu J: Tumor associated macrophage $\mathrm{x}$ cancer cell hybrids may acquire cancer stem cell properties in breast cancer. PLoS One 7: e41942, 2012.

10. Lu X and Kang Y: Cell fusion as a hidden force in tumor progression. Cancer Res 69: 8536-8539, 2009.

11. Lu X and Kang Y: Cell fusion hypothesis of the cancer stem cell. Adv Exp Med Biol 714: 129-140, 2011.

12. Bjerkvig R, Tysnes BB, Aboody KS, Najbauer $\mathbf{J}$ and Teris AJ: Opinion: the origin of the cancer stem cell: current controversies and new insights. Nat Rev Cancer 5: 899-904, 2005.

13. Pawelek JM and Chakraborty AK: Fusion of tumour cells with bone marrow-derived cells: a unifying explanation for metastasis. Nat Rev Cancer 8: 377-386, 2008.

14. Soleimani M and Nadri S: A protocol for isolation and culture of mesenchymal stem cells from mouse bone marrow. Nat Protoc 4 : 102-106, 2009

15. Köhler G and Milstein C: Continuous cultures of fused cells secreting antibody of predefined specificity. Nature 256: 495-497, 1975.
16. Yang MH, Chang SY, Chiou SH, Liu CJ, Chi CW, Chen PM, et al: Overexpression of NBS1 induces epithelial-mesenchymal transition and co-expression of NBS1 and Snail predicts metastasis of head and neck cancer. Oncogene 26: 1459-1467, 2007.

17. Duelli D and Lazebnik Y: Cell fusion: a hidden enemy? Cancer Cell 3: 445-448, 2003.

18. Gerling M, Nousiainen K, Hautaniemi S, Krüger S, Fritzsche B, Homann N, et al: Aneuploidy-associated gene expression signatures characterize malignant transformation in ulcerative colitis Inflamm Bowel Dis 19: 691-703, 2013.

19. Luzzo KM, Wang Q, Purcell SH, Chi M, Jimenez PT, Grindler N, et al: High fat diet induced developmental defects in the mouse: oocyte meiotic aneuploidy and fetal growth retardation/brain defects. PLoS One 7: e49217, 2012.

20. Hanna MO, Zayed NA, Darwish H and Girgis SI: Asynchronous DNA replication and aneuploidy in lymphocytes of hepatocellular carcinoma patients. Cancer Genet 205: 636-643, 2012.

21. Pfau SJ and Amon A: Chromosomal instability and aneuploidy in cancer: from yeast to man. EMBO Rep 13: 515-527, 2012.

22. Stein-Werblowsky R and Ablin RJ: Consideration of the possible etiology of prostatic cancer. II International Congress of Andrology, Tel Aviv, Israel. Israel J Med Sci 17: abs. 743, 1981.

23. Fan F, Samuel S, Evans KW, Lu J, Xia L, Zhou Y, et al: Overexpression of Snail induces epithelial-mesenchymal transition and a cancer stem cell-like phenotype in human colorectal cancer cells. Cancer Med 1: 5-16, 2012

24. Gavert N and Ben-Ze'ev A: Epithelial-mesenchymal transition and the invasive potential of tumors. Trends Mol Med 14: 199-209, 2008

25. Horikawa T, Yoshizaki T, Kondo S, Furukawa M, Kaizaki Y and Pagano JS: Epstein-Barr virus latent membrane protein 1 induces Snail and epithelial-mesenchymal transition in metastatic nasopharyngeal carcinoma. Br J Cancer 104: 1160-1167, 2011.

26. Jordà $\mathrm{M}$, Olmeda $\mathrm{D}$, Vinyals $\mathrm{A}$, Valero $\mathrm{E}$, Cubillo $\mathrm{E}$, Llorens $\mathrm{A}$, et al: Upregulation of MMP-9 in MDCK epithelial cell line in response to expression of the Snail transcription factor. J Cell Sci 118: 3371-3385, 2005

27. Parsons JT, Zetter B and Mohla S: Shifting paradigms in tumor metastasis: challenges and opportunities. Cancer Biol Ther 1: 582-585, 2002.

28. Goldstein RH, Weinberg RA and Rosenblatt M: Of mice and (wo)men: mouse models of breast cancer metastasis to bone. J Bone Miner Res 25: 431-436, 2010.

29. Sasai K, Sukezane T, Yanagita E, Nakagawa H, Hotta A, Itoh T and Akagi T: Oncogene-mediated human lung epithelial cell transformation produces adenocarcinoma phenotypes in vivo. Cancer Res 71: 2541-2549, 2011. 